\title{
The Semantic and Phonological Priming in Written Chinese Word Recognition
}

\author{
Ran Wei \\ School of Foreign Studies, Jiangsu Normal University, Xuzhou, China \\ E-mail: weiran@jsnu.edu.cn
}

Received: January 11, 2022 Accepted: February 13, 2022 Published: February 17, 2022

doi:10.5296/ijl.v14i1.19460

URL: https://doi.org/10.5296/ijl.v14i1.19460

\begin{abstract}
Semantic priming refers to a facilitation of responding speed due to the presentation of a semantically related word, while, similarly, phonological priming refers to improved recognition of a target word preceded by a word sharing the same phonological feature. Even though both of these priming effects were proved to be effective in lexical processing, it is still uncertain about whether there is difference between semantic priming effect and phonological priming effect in visual word recognition. This study focused on semantic and phonological priming effects in reading two-character Chinese words. With the lexical decision task, 18 native Mandarin speakers voluntarily took part in the experiment. It was shown that the responding speed to the target word preceded by semantic primes was faster than that preceded by phonological primes than that preceded by unrelated control primes. In other words, there are positive semantic and phonological priming effects in reading Chinese, and the effect of semantic priming is stronger than that of phonological priming. The findings supported dual-route model in lexical processing and shed more light on the studies concerning semantic and phonological priming effects in reading Chinese words.
\end{abstract}

Keywords: Chinese word recognition, Semantic priming, Phonological priming, Dual-route model

\section{Introduction}

It is widely accepted that the semantic association between a target word (e.g., nurse) and another word (e.g., doctor) preceding it would speed up its lexical processing. In other words, people's responding speed to the target word is faster when it is preceded by a semantically related word than when it is preceded by an unrelated control word. We regard the semantically related word preceding the target word as the semantic prime. It is believed that the semantic association between the prime and its target word activates the target word, promoting its processing or recognition (Neely, 1991; McNamara, 2005). Similarly, the 
responding speed to the target word is also likely to be faster when it is preceded by a word sharing the same phonological feature (e.g., homophone or semihomophone) than when it is preceded by a totally unrelated word (Zhou \& Wilson-William, 1999). The phonological priming probably occurs because the sound consistency between the prime and the target word facilitates the lexical recognition of the target word. The phonological effect in lexical semantic access has been reported in Stroop paradigm (Spinks \& Liu, 2000; Guo et al., 2004). But is there any difference between semantic priming effect and phonological priming effect in visual word recognition? Zhou \& Marslen-Wilson (2000) compared the relative time course of semantic and phonological activation in reading two-character Chinese words and reported that semantic primes had a strong effect on words recognition while the phonological primes failed to have either positive or negative effects on the target words. Tse and Yap (2018) suggested that Chinese skilled readers rely more on semantic information than phonological information when they are processing visually presented words. However, Williams and Bever (2010) found that educated Chinese speakers used both semantic and phonetic paths to character encoding, different from the above claim of semantic priming effect over phonological priming effect on character encoding. With regard to the semantic priming effect, Peng and colleges (2020) claimed that semantic activation in decoding in Chinese reading comprehension is insufficient for Chinese skilled readers, more complex syntactic skills are also needed. It is noteworthy that there is inconsistency in documented literature on the semantic/phonological activation in Chinese words recognition, this study therefore intends to focus on the same research questions as Zhou \& Marslen-Wilson (2000) investigated (i.e., Are there any semantic and/or phonological priming effects in reading two-character Chinese words? And if so, which priming effect, semantic or phonological, is stronger in reading Chinese?) to shed more light on the studies concerning semantic and phonological priming effects in reading Chinese words.

Two predictions are made before the experiment, that is, 1) both semantic and phonological primes have positive effect in Chinese word recognition compared to the unrelated control primes, and 2) the effect of semantic primes is stronger than that of phonological primes in reading Chinese, as shown in Figure 1. In other words, people respond to the target word preceded by its meaning/sound related word faster than to the word preceded by totally unrelated word and people's reaction speed to the word preceded by the meaning related word is faster than that preceded by word with the same lexical tone. Suppose a target word (e.g., 记忆ji4yi4, memory) is preceded by its semantic prime (e.g., 思维si1wei2, thinking) or by its phonological prime (e.g., 技艺ji4yi4, skill) or by a totally unrelated control prime (e.g., 朴素pu3su4, plain), the reaction time to the word of '记忆ji4yi4, memory' is longer when it is preceded by a totally unrelated control prime ‘朴素pu3su4, plain' compared to its semantic prime of '思维si1wei2, thinking' and its phonological prime of ‘技艺ji4yi4, skill’. It is probably due to the semantic or phonological association between the target word and its priming words which activates the target word and facilitates its lexical processing. In addition, the reaction time to the word of '记忆ji4yi4, memory' is shorter when it is preceded by its semantic prime of '思维si1wei2, thinking' than when it is preceded by its phonological prime of '技艺ji4yi4, skill'. It is attributed to the fact that logographic languages like Chinese closely connect the visual word recognition with semantics but comparatively split it away 


\section{Macrothink}

International Journal of Linguistics

ISSN 1948-5425

2022, Vol. 14, No. 1

from phonology. If we draw the bar graph for reaction time, the bar for the unrelated control primes should be the highest among the three and the bar for semantic primes should be shorter and lower than that for phonological primes. That is to say, both semantic primes and phonological primes have positive effects in reading Chinese but the semantic priming effect is stronger than phonological priming effect. The following figure shows the predicted results.

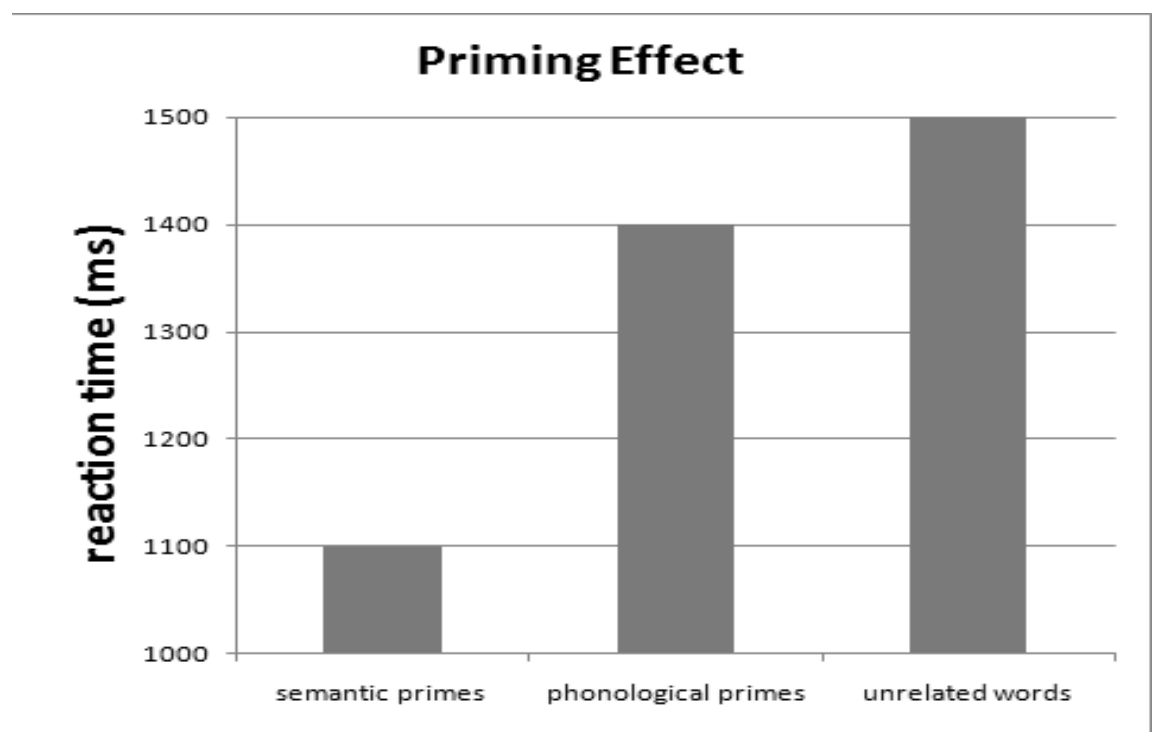

Figure 1. Predicted reaction time of target word under three priming conditions

To justify the proposed research predictions, two-character target words and priming words (semantic, phonological and unrelated control) were firstly selected for the experiment. Native Mandarin speakers were invited to attend the experiment and asked to press computer button showing their judgment about whether the word in the computer screen is a real word or not. After the experiment was finished and all the data were collected, a bar graph reflecting reaction time for semantic primes, phonological primes, and unrelated control primes was drawn to show the priming effect difference in reading Chinese.

\section{Methods}

\subsection{Participants}

18 L1 Mandarin speakers (5 males and 13 females) voluntarily attended the experiment. All of them were born in mainland China. Some studied abroad while others currently working in mainland. Their ages stemmed from 22 to 37 (i.e., they are 22, 26, 27, 28, 28, 29, 29, 29, 32, $33,34,34,35,35,36,36,37,37$ years old, respectively) with the average age 31.5 years old. Their eyesight or corrected visions were normal.

\subsection{Stimuli}

Based on The Top 3000 words in Modern Chinese 'xian dai han yu san qian chang yong ci biao’ (He \& Li, 1987), 60 target words were selected. Every target word (e.g., 记忆ji4yi4, memory) has two priming words, namely semantic prime (e.g., 思维si1wei2, thinking) and 
phonological prime (技艺ji4yi4, skill), and one unrelated control prime that has no relation either with the meaning or the sound of the target word (e.g., 朴素pu3su4, plain). Two postgraduates from Chinese majors assisted in checking the semantic primes so as to guarantee the semantic association between each target word and its semantic prime. Every phonological prime (i.e., homophone) shares the same lexical tone with its target word. Since all the target words and priming words were selected from the top 3000 modern Chinese words and the word frequencies are comparatively high, it ensures the participants' familiarity with all of them. Besides, the selection of the words also considered the visual complexity (i.e., strokes of a Chinese character) and the difference of numbers of strokes in the primes for each target word was controlled within 10 so as to avoid the possible interference from the word orthography. In addition, another 60 pairs of word-nonword fillers were selected so as to eliminate or diminish the strategies participants could use in the experiment. The following table shows an example of the prepared stimuli.

Table 1. Stimuli example

\begin{tabular}{cccc}
\hline Target word & Semantic prime & Phonological prime & Unrelated control prime \\
\hline 记忆 & 思维 & 技艺 & 朴素 \\
\hline ji4yi4 & si1wei2 & ji4yi4 & pu3su4 \\
\hline memory & thinking & skill & plain \\
\hline
\end{tabular}

In order to familiarize participants in the experiment, 20 pairs of words as practice items including 10 pairs of word-word items and 10 pairs of word-nonword items had been provided as well. Finally, 20 pairs of practice items, 60 targets words with relevant 60 semantic and 60 phonological primes and 60 totally unrelated control primes, and 60 word-nonword pairs were prepared before the experiment.

\subsection{Procedure}

After the preparation for all the stimuli, I logged into the online experiment design website ibex farm http://spellout.net/ibexfarm to design the priming experiment. First, I wrote an introduction statement in Chinese in a plain text file based on the template and then edited all the prepared words and non-words in the excel template to make ibex code. The time duration for each priming word on the screen was $250 \mathrm{~ms}$ and the corresponding target word presented immediately after the prime on the computer screen for participants to judge whether it is a real word or a non-word. Once the participants pressed the button, the time duration for the appearance of next pair of priming word was $1250 \mathrm{~ms}$. I then uploaded the introduction file and the code file into ibex and run the experiment first to check whether it worked well. After all these were completely done, I sent the ibex link http://spellout.net/ibexexps/carolweiran/priming/experiment.html to the participants and asked them to do the experiment on their computers. Since ibex can automatically do Latin-square design, each participant who received the ibex link would randomly get 60 target words with their corresponding priming words $(20$ with semantic or 20 with 
phonological or 20 with unrelated control words) and 60 word-nonword pairs. A pair of word-word or word-nonword items appeared one after another, and then the second word in the pair was the one for participants to make judgment. Participants were asked to press " 1 " if it is a real Chinese word and press " 2 " if it is a non-word in Chinese. After their decision, the second pair of word-word or word-nonword items was presented on the screen. Soon after sending the online experiment link to each of the participants, I provided a brief introduction about this experiment including the procedures and time duration. Besides, I also emphasized that they had to do the experiment on their computers with keyboard buttons instead of their cell phones or mouse and they had to make their judgment as quickly and correctly as possible. After a participant finished his/her experiment, I sent the link to another one and did the same instruction as well.

\section{Results}

When all the participants finished their experiments and all data were received automatically by ibex, I saved them firstly in plain text files and then input into excel files to do the data analysis. Only real words with correct judgment (i.e., the related word people say it is a real word) can be used to calculate the reaction time. All the practice items, non-words, and real words with wrong judgments were deleted. Next, for each participant, I manually selected all the data of real words with correct decision and then draw a scattered plot to show the whole distribution of the reaction time for all the semantic, phonological, and unrelated control primes and then visually checked if there were any obvious outliners (i.e., extremely higher or lower than the others) ready to be removed. Provided that some items are obvious outliners, they have to be deleted to avoid their interference to the final average score. For instance, if the reaction time for all the real words is around $1000 \mathrm{~ms}$ to $2000 \mathrm{~ms}$ but two of them are over $6000 \mathrm{~ms}$ and one of them are below $300 \mathrm{~ms}$, then the three items should be deleted to avoid the average interference. Then the reaction time for semantic primes, phonological primes, and unrelated control primes were averaged respectively. The bar graph below shows the different reaction time among them.

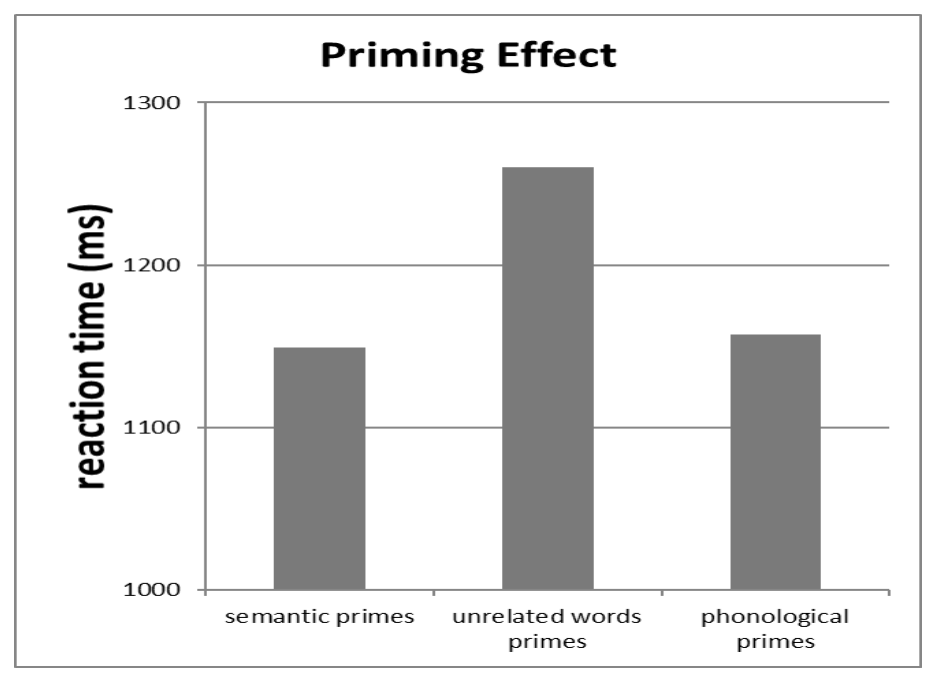

Figure 2. Averaged reaction time of target words under three priming conditions 
It is shown from the above figure in the result section that the bar for unrelated control primes is higher than that for phonological primes than that for semantic primes. People respond to the target word preceded by semantic primes (around $1148 \mathrm{~ms}$ ) faster than that preceded by phonological primes (around $1157 \mathrm{~ms}$ ) than that preceded by unrelated control primes (around $1260 \mathrm{~ms}$ ). What's more, statistical analysis further convinced that the responding speed in semantic primes condition was statistically significantly faster than that in unrelated control primes condition $(\mathrm{p}=0.033)$ and the facilitation of responding speed in phonological primes condition was marginally statistically significant $(\mathrm{p}=0.073)$. however, the responding speed in both semantic and phonological primes condition remained the same with no statistical significance $(\mathrm{p}=0.406)$. It ultimately supports the previous prediction that both semantic and phonological primes have positive effect in Chinese word recognition compared to the unrelated control primes. In other words, semantic association and phonological similarity of homophones do facilitate the lexical processing of the target word, contribute to its activation and bring about positive priming effect which promotes the recognition of the target word.

For the finding that there is a positive semantic priming effect in reading Chinese, it is probably because the visual input from orthography directly activates the word-form representation in mental lexicon, ultimately realizing semantic activation. And for another finding that the effect of phonological priming is also positive, the result is not in consistency with Zhou \& Marslen-Wilson (2000). Zhou \& Marslen-Wilson (2000) compared the relative time course of semantic and phonological activation in reading two-character Chinese words and found that semantic primes had a strong effect on words recognition while the phonological primes failed to have either positive or negative effects on the target words. Zhou (1997) also reported the same limited effects of phonological primes in semantic activation of Chinese word. However, the findings of present study presented the opposite effect of phonological priming in Chinese words recognition. The different stimuli selection can probably explain the different result in these two studies. It is easy to realize direct access from word form to word semantic meaning for high proficient words, while for those words in low proficiency, phonology plays a mediated role in semantic access. The reason behind the positive phonological priming effect in present study is probably due to the fact that the phonological primes share the same lexical tone with the target words, which make Chinese lexical processing to be phonological-mediated indirect access. In other words, the lexical semantic access relies on the mediation of phonology, verifying dual-route model of lexical processing. For instance, the target word '记忆ji4yi4, memory' has a phonological prime ‘技 艺ji4yi4, skill'. When the target word '记忆ji4yi4, memory' is preceded by the phonological prime '技艺ji4yi4, skill', it not only activates the sound of the target word '记忆ji4yi4, memory' but also facilitates its activation because of its mediated effects. Additionally, P200 is a criterion for phonological processing (Kong et al., 2010). According to the findings from an ERP study, P200 is likely to be related with phonological activation in an early stage and direct semantic processing and lexical access also probably relate with P200 (Liu et al., 2011). To summarize, the experiment results therefore support the previous research prediction that semantic priming has a positive effect in Chinese word recognition but also confirm the facilitative role of phonological priming in visual lexical processing. 


\section{Conclusions}

This study focused on semantic and phonological priming effects in written Chinese word recognition with lexical decision task. It was found that participants responded to the target word preceded by semantic primes faster than that preceded by phonological primes than that preceded by unrelated control primes. However, the facilitation of responding speed under both semantic and phonological primes remained the same. It ultimately supports the previous prediction that both semantic and phonological primes have positive effect in Chinese word recognition compared to the unrelated control primes. In other words, semantic association and phonological similarity of homophones do facilitate the lexical processing of the target word, contribute to its activation and bring about positive priming effect which promotes the recognition of the target word.

For the research limitations, there were only 18 participants with unequal gender distribution (i.e., male to female 5: 13) and only behavioral experiment rather than EEG/ERP experiment was conducted in this experiment. In addition, the nature of different experiments and stimuli (e.g., stimulus onset asynchrony (SOA) and phonological selection such as homophone or semihomophone) could vary in certain range. All these factors could influence the final results of the experiment. Therefore, more experiments should be conducted in the future to further justify the semantic and phonological priming effect in visual Chinese word recognition.

\section{Acknowledgements}

This work was supported by The Project of Philosophy and Social Science Research in Colleges and Universities in Jiangsu Province (grant No. 2017SJB0948); Jiangsu Normal University Doctoral Teachers Scientific Research Support Project (grant No. 19XFRX001).

\section{References}

Guo, T. M., Peng, D. L., Qi, Z. Q., \& Xu, L. (2004). Automatic phonological activation and its role in access the meaning of Chinese character (yu yin de zi dong ji huo ji qi zai han zi yu yi tong da zhong de zuo yong). Psychological Exploration, 1, 31-33.

He, K. K., \& Li, D. K. (1987). The top 3000 words in modern Chinese (xian dai han yu san qian chang yong ci biao). Beijing: Beijing Normal University Press.

Kong, L. Y., Zhang, J. X., Kang, C. P. Du, Y. C., Zhang, B., \& Wang, S. P. (2010). P200 and phonological processing in Chinese word recognition. Neuroscience Letters, 473(1), 37-41. https://doi.org/10.1016/j.neulet.2010.02.014

Liu, B. L., Jin, Z., Qing, Z., \& Wang, Z. (2011). The processing of phonological, orthographical, and lexical information of Chinese characters in sentence contexts: An ERP study. Brain Research, 1372(4), 81-91. https://doi.org/10.1016/j.brainres.2010.11.068

McNamara, T. S. (2005). Semantic priming: Perspectives from memory and word recognition. New York: Psychology Press.

Neely, J. H. (1991). Semantic priming effects in visual word recognition: A selective review 
of current findings and theories. Basic Processes in Reading, 11, 264-336. https://doi.org/10.4324/9780203052242-12

Peng, P., Lee, K., Luo, J., Li, S., Joshi, R. M., \& Tao, S. (2020). Simple view of reading in Chinese: A one-stage meta-analytic structural equation modeling. Review of Educational Research, 91(1), 3-33. https://doi.org/10.3102/0034654320964198

Spinks, J. A., \& Liu, Y. (2000). Reading Chinese character for meaning: The role of phonological information. Cognition, $\quad 76, \quad 1-11$. https://doi.org/10.1016/S0010-0277(00)00072-X

Tse, C. S., \& Yap, M. J. (2018). The role of lexical variables in the visual recognition of two-character Chinese compound words: A megastudy analysis. Quarterly Journal of Experimental Psychology, 71(9), 2022-2038. https://doi.org/10.1177/1747021817738965

Williams, C., \& Bever, T. (2010). Chinese character decoding: a semantic bias?. Reading and Writing, 23(5), 589-605. https://doi.org/10.1007/s11145-010-9228-0

Zhou, X. L. (1997). Limited phonological effect in semantic activation (yu yi ji huo zhong yu yin de you xian zuo yong). In R. L. Peng, H. Shu, \& H. Z. Chen (Eds.), Chinese cognitive research (pp. 159-184). Jinan, Shandong: Shandong Education Press.

Zhou, X. L., \& Marslen-Wilson, W. (2000). The relative time course of semantic and phonological activation in reading Chinese. Journal of Experimental Psychology: Learning, Memory, and Cognition, 26(5), 1245-1265. https://doi.org/10.1037//0278-7393.26.5.1245

Zhou, X. L., \& Wilson-William, M. (1999). Phonology, orthography and semantic activation in reading Chinese. Journal of Memory and Language, 41(4), 579-606. https://doi.org/10.1006/jmla.1999.2663

\section{Copyrights}

Copyright for this article is retained by the author(s), with first publication rights granted to the journal.

This is an open-access article distributed under the terms and conditions of the Creative Commons Attribution license (http://creativecommons.org/licenses/by/4.0/) 